\title{
Study of the Efficiency and Workflow of Femtosecond Laser-Assisted Cataract Surgery in a Spanish Public Hospital
}

Jacinto Villavilla-Castillo

Hospital Regional Universitario de Malaga

Marina Rodríguez Calvo de Mora ( $\square$ marocalmo@gmail.com )

Hospital Regional Universitario de Malaga https://orcid.org/0000-0002-5513-8790

Cristina Pérez-Casaseca

Hospital Regional Universitario de Malaga

Emilio Espejo de-los-Riscos

Hospital Regional Universitario de Malaga

Gustavo Fernandez Baca

Hospital Regional Universitario, Málaga

Ildefonso Fernández-Baca-Casares

Hospital Regional Universitario de Malaga

David P. Piñero-Llorens

Universitat d'Alacant

Research article

Keywords: Femtosecond Laser-Assisted Cataract Surgery, cataract surgery, phacoemulsification, operation room workflow, public setting

Posted Date: May 24th, 2019

DOI: https://doi.org/10.21203/rs.2.9794/v1

License: (c) (1) This work is licensed under a Creative Commons Attribution 4.0 International License.

Read Full License

Version of Record: A version of this preprint was published at Journal Français d'Ophtalmologie on July 1st, 2021. See the published version at https://doi.org/10.1016/j.jfo.2021.01.030. 


\section{Abstract}

Background: To assess the time-efficiency of a designated operation room (OR) workflow in the introduction of Femtosecond laser-assisted cataract surgery (FLACS, LenSx, Alcon®). The study was carried out in a public hospital with high volume of procedures. Methods: We performed this prospective, controlled, surgical intervention study in the Ophthalmology department of a tertiary referral Spanish public hospital. A total of 167 eyes were enrolled, including 62 eyes undergoing conventional phacoemulsification surgery. In phase I, patients were assigned either to FLACS-I $(n=63)$ or conventional phacoemulsification surgery $(n=62)$. One surgeon operated the Femto-second laser, another finished the procedure, whereas another performed a conventional phacoemulsification. In the second phase (FLACSII), all the surgeries were FLACS $(n=42)$. A surgeon performed the FLACS procedure and two different surgeons completed the surgeries in separated ORs. Surgical and roll-over times of all the patients were recorded. Results: Preparation time was statistically significant lower in FLACS-I and FLACS-II $(p<0.001)$ whereas the time of the cataract procedure per se was higher in FLACS-II compared to conventional phacoemulsification $(p=0.03)$. Phacoemulsification energy was higher in FLACS-II compared to FLACS-I $(p=0.01)$ whereas laser-related surgical time was lower $(p=0.001)$. Surgical complications and total surgical time showed no statistically significant differences between all three groups. Conclusions: This study suggests a time-efficient and suitable workflow model for FLACS considering the specific requirements and restrictions of a saturated public hospital. Even so we have shown that FLACS procedure does not take longer than conventional phacoemulsification following a detailed planning for the OR workflow. Besides, our data reflect an improvement surgical times in FLACS with ongoing experience. Trial registration: NCT03931629 (retrospectively registered)

\section{Background}

Cataract surgery is the most common surgical intervention conducted worldwide. ${ }^{1}$ It is also one of the most cost-effective ophthalmological procedure, leading to great improvements in patients' quality of life. ${ }^{2}$ Technological advances have allowed the introduction of substantial improvements in cataract surgery techniques and outcomes over the past twenty years, including the evolution from intracapsular surgery to phacoemulsification, the development of microincision surgery, the introduction of femtosecond laser technology to assist during the surgical procedure, and the development of sophisticated models of intraocular lenses (IOLs) allowing maximum levels of postoperative spectacle independence. ${ }^{3-5}$

One of the most recent advances is the introduction and development of femtosecond laser assisted cataract surgery (FLACS). With the precision of currently available platforms for FLACS, some sections of the cataract surgery can be safely performed, with a completion of the surgical procedure with some conventional steps, such as nucleus and mass aspiration, IOL injection and stromal hydration. Several authors have claimed that FLACS outperforms traditional phacoemulsification surgery in terms of safety, accuracy and reproducibility. ${ }^{6,7}$ Likewise, FLACS has been shown to be especially useful in certain 
circumstances, such as in shallow anterior chamber eyes, ${ }^{5}$ very hard cataracts, ${ }^{8}$ infantile cataracts (easier to perform posterior capsulotomy), ${ }^{5,9,10}$ low endothelial cell counts ${ }^{11-13}$ and unstable zonules. ${ }^{5,8}$ However, recent meta-analyses have not shown statistically significant differences between FLACS and conventional phacoemulsification. ${ }^{1,3}$

The potential advantages of FLACS over conventional procedures include lower postoperative residual refractive error possibly due to a more optimal creation of incisions ${ }^{14,15}$ and the possibility of making additional arcuate incisions to control astigmatism, ${ }^{5}$ and less corneal endothelial cell damage due to more reduced effective phaco time. 5,16,17 Likewise, the femtosecond laser technology allows the creation of a precise capsulotomy, facilitating a perfect centration of the IOL into the capsular bag and then avoiding the "lens tilt" phenomenon, which can lead to refractive errors despite correct biometric calculations. ${ }^{18-21}$

Concerning the disadvantages of FLACS, one of the most relevant is the considerable initial investment required to acquire the laser platform, ${ }^{22}$ with some concerns about the cost-effectiveness of the technique. ${ }^{23}$ Furthermore, although there are advantages of FLACS over conventional phacoemulsification in terms of safety and postoperative refractive error, complications with conventional phacoemulsification surgery are very uncommon, compared to FLACS. ${ }^{3}$ In contrast, a learning curve of about 100 cataracts has been described for FLACS, which is significantly shorter in ophthalmologists performing refractive surgery compared to conventional phacoemulsification. ${ }^{24,25}$

Most of scientific evidence on postoperative outcomes and safety of cataract surgery using femtosecond laser and subsequent phacoemulsification to this date are based on studies conducted at private centres, ${ }^{1}$ but in recent years, growing scientific evidence of the use of FLACS in public hospitals and how its introduction may affect to the efficiency of the public system is being published. ${ }^{26-28}$

Besides the assessment of safety of FLACS, the aim of our study was also to evaluate the surgical room workflow and its efficiency in a public health center with a large volume of surgeries ( 21 cataracts per day, on average) in comparison with the conventional procedure. It should be remarked that the same surgeons perform the femtosecond-laser part of the surgery as well as the procedures in the control group following the conventional protocol to minimize the potential bias due to the "surgeon factor". All of the them were consultants with wide experience both in conventional phacoemulsification and refractive surgery.

To our knowledge, this is the first study with the aim of evaluating FLACS workflow and roll over times in a public hospital. The results of this study could provide data that would help to clarify whether investing in femtosecond technology in public health for cataract surgery can be justified.

\section{Methods}


Patient recruitment. This single-center, prospective, consecutive, interventional, and non-randomized study was conducted at the Department of Ophthalmology of the Regional University Hospital of Málaga (Spain) from February to June 2016. Patients were recruited in this clinical setting during routine activity. Inclusion criteria for the study were signed informed consent, patients of more than 18 years old, presence of visually significant cataract (best corrected visual acuity worse than $0.4 \log M A R$ ), and possibility of performing cataract surgery under local anaesthesia (topical, sub-tenon or retrobulbar). Exclusion criteria were patients who refused to give their consent, traumatic, congenital, luxated, subluxated cataracts, or with manifest zonular weakness, vitrectomized patients, small pupils (mydriasis $6 \mathrm{~mm}$ ), narrow palpebral fissure, advanced optic nerve glaucomatous excavation, irregular astigmatism, significant corneal opacities, severe ocular surface disease, keratoconus, stromal scarring or edema and patients requiring general anaesthesia for the intervention. Patients who complied with the inclusion and exclusion criteria mentioned above signed a specific informed consent for the study and were nonrandomly assigned to the FLACS (femtosecond laser-assisted cataract surgery) or MANUAL (manual or conventional phacoemulsification surgery) group. We checked that both groups were comparable in terms of age and gender.The three surgeons involved in the study were highly experienced consultants in performing conventional cataract surgery (from 15 to 20 years of experience, performing from 500 to 800 phacoemulsifications yearly) and refractive surgery (10 to 15 years of experience, performing from 200 to 500 refractive procedures).This study has been carried out following the Declaration of Helsinki and Good Clinical Practices guidelines. Personal data was processed according to guidelines established by the Spanish Law of Data Protection.

Study design.This study was composed of two phases and was carried out by three consultant surgeons. No resident or trainee was involved in the performance of the surgeries. In Phase I, one of the three surgeons operated the LensX®, another performed the FLACS surgery (FLACS I) in one operating room (OR1), while the third surgeon performed the conventional phacoemulsification procedure (MANUAL) in another operating room (OR2). On subsequent days, the surgeons rotated between LensX®, FLACS and MANUAL surgery (Figure 1). All the settings for the Infiniti ${ }^{\circledR}$ and the LensX® were the same for all surgeons (see below). The cataracts included in the FLACS I group were presumably straightforward, as they were part of the learning curve for the surgeons and were evaluated by consultant ophthalmologists (it must be considered that the maximum best-corrected visual acuity [BCVA] for being eligible for cataract surgery in the Andalusian public System is $0.4 \log$ MAR). Patients with easily accessible sockets and good patient collaboration were included. Eligible cataracts ranged from nuclear opacities from NC1 to NC4, cortical opacities from $\mathrm{C} 1$ to $\mathrm{C} 4$ and posterior subcapsular from P1 to P4, according to the Lens Opacities Classifications System (LOCSIII); with good pupil dilation (minimum $8 \mathrm{~mm}$, that was measured in the slit lamp as described by $\mathrm{Ho} \mathrm{et} \mathrm{al}^{29}$ ) after the standard dilation protocol, i.e., instillation of tropicamide $1 \%$ and fenylephrine $10 \%$ eye-drops in the conjunctival sac, three times, each time separated by 10 minutes. Fuchs endothelial dystrophy and white cataracts were excluded. To ensure the comparability among groups, cataracts included in the MANUAL group were selected with similar characteristics.In phase II, one of the three surgeons operated the LensX®, while the other two surgeons completed the procedures in two different ORs (FLACS II)(Figure 2). Surgeons rotated in subsequent 
days. The FLACS II group included patients with harder cataracts (nuclear opacities from NC4 to NC6, cortical opacities from C3 to C5 and posterior subcapsular from P3 to P5, according to the LOCSIII), smaller orbits, suboptimal mydriasis (from 6 to $8 \mathrm{~mm}$, measured in the slit lamp as mentioned before), Fuchs endothelial dystrophy and white cataracts. (Images 1-3). Therefore, this group included the whole range of cataract surgeries that are commonly performed at a public hospital.

Procedures.Prior to surgery, the routine dilation protocol (instillation of tropicamide $1 \%$ and fenylephrine $10 \%$ eye-drops in the conjunctival sac, three times, each time separated by 10 minutes), preoperative disinfection (cleaning of eyelid and periorbital region with $10 \%$ povidone iodine and instillation of povidone iodine $5 \%$ in in the conjunctival sac) and placement of sterile dressing were performed as usual. Conventional phacoemulsification was performed using the divide-and-conquer technique through a 2.2$\mathrm{mm}$ clear corneal incision and the platform Infiniti ${ }^{\circledR}$ (Alcon ${ }^{\circledR}$, Fort Worth, Texas, USA). FLACS was performed using the LenS $x \circledR$ platform (Alcon ${ }^{\circledR}$ Fort Worth, Texas, USA). The femtosecond laser was placed in a room adjacent to the OR, complying with current sterility standards mentioned above. Patients were transported from the laser room to the OR in an ophthalmological surgical chair. After completing the laser-assisted procedures (capsulorrhexis, nucleus fragmentation, main and accessory incisions), the patient entered to the $\mathrm{OR}$ where the phacoemulsification and aspiration of the nucleus and cortical masses, implantation of the IOL (AcrySof $®$ IQ Monofocal IOL, Alcon, Fort Worth, Texas, USA), and hydration of surgical borders were performed. The ophthalmic viscosurgical device used in all cases was DuoVisc ${ }^{\circledR}$ Alcon, Fort Worth, Texas, USA). Prophylactic $0.1 \mathrm{~mL}$ of cefuroxime $(1 \mathrm{mg} / 0.1 \mathrm{~mL})$ was injected in the anterior chamber at the end of the surgery. The postoperative follow-up was performed according to usual protocols.

Settings.All the settings for the Infiniti ${ }^{\circledR}$ and the Lens $X ®$ were the same for all surgeons. The Infiniti ${ }^{\circledR}$ settings were the following: for the pre-phaco, $80 \mathrm{cmH} 20$ of irrigation, $150 \mathrm{mmHg}$ of vacuum and 25 $\mathrm{mL} / \mathrm{min}$ of flow aspiration; for sculpt, $80 \mathrm{cmH} 20$ of irrigation, $70 \%$ of phaco energy and $95 \%$ of torsional amplitude, $50 \mathrm{mmHg}$ of vacuum and $25 \mathrm{~mL} / \mathrm{min}$ of flow aspiration; for segment removal: $110 \mathrm{cmH} 20$ of irrigation, 90 of torsional amplitude, $400 \mathrm{mmHg}$ of vacuum and $25 \mathrm{~mL} / \mathrm{min}$ of flow aspiration; for the epinucleus removal: $110 \mathrm{cmH} 20$ of irrigation, $230 \mathrm{mmHg}$ of vacuum and $25 \mathrm{~mL} / \mathrm{min}$ of flow aspiration; for the irrigation/aspiration: $65 \mathrm{cmH} 20$ of irrigation, $450 \mathrm{mmHg}$ of vacuum and $25 \mathrm{~mL} / \mathrm{min}$ of flow aspiration; for the visco $65 \mathrm{cmH} 20$ of irrigation, $500 \mathrm{mmHg}$ of vacuum and $30 \mathrm{~mL} / \mathrm{min}$ of flow aspiration. Continous Ozil was used throughout the phacoemulsification. The settings for LensX® were the following. For the capsulotomy: diameter $5 \mathrm{~mm}$, energy $5 \mu \mathrm{J}$, spot separation $4 \mu \mathrm{m}$, layer separation $3 \mu \mathrm{m}$, delta up (lens anterior offset) $300 \mu \mathrm{m}$, delta down (lens posterior offset) $300 \mu \mathrm{m}$. A three-plane incision was used for primary incision at 135 degrees (posterior depth 40,80 and $130 \%$, respectively). Secondary incision was performed at 45 degrees. For both incisions, energy used was $4 \mu \mathrm{J}$ and spot and layer separation was $4 \mu \mathrm{m}$. The laser pattern for fragmentation was a hybrid combination between chop (two cuts) and cylinder.

Measurements.The surgical and roll-over times for both conventional phacoemulsification and FLACS were recorded in a spreadsheet including: start time of patient preparation, start time of anesthesia, 
surgery start time, surgery end time, time needed to transfer the patient to resting area, and time of discharge. Specifically, for patients undergoing FLACS, the sum of the preparation time and the femtosecond laser time was calculated as well as the time between the performance of femtosecond laser procedures and the beginning of phacoemulsification surgery, and the time ranging from the moment when the patient entered to the femtosecond laser room to the moment where the patient left after the end of the surgery. Likewise, the following parameters were calculated in all groups: time from the insertion of blepharostat to its removal after ending the surgical procedure, time from the moment the patient entered the OR to the moment the patient left the OR after finishing the surgery, and time from the admission of the patient to the surgical area to the discharge once the intervention was finished. Likewise, mean phacoemulsification energy used in each procedure was also recorded (CDE: cumulative dissipated energy). Finally, intraoperative complications were also recorded, including loss of suction, loss of fixation/follow-up and incomplete capsulorrhexis for the femtosecond laser-assisted stage, and incomplete rexis, capsular rupture, $\mathrm{IOL}$ dislocation and incisions without coaptation for the phacoemulsification stage.

Statistical analysis.A descriptive analysis of the variables studied including mean, standard deviation, maximum and minimum. To analyse the differences between FLACS and MANUAL groups in terms of the continuous quantitative variables, the unpaired Student $t$ test was applied whenever the normality condition was satisfied (verified with the Shapiro-Wilk test). Otherwise, the corresponding non-parametric test was used (Mann-Whitney test). Comparisons of categorical variables were performed using the Chisquare test. A homogeneity analysis was performed between groups in terms of age and gender to ensure the comparability of groups. All these statistical analyses were performed using R software version 3.2.1 (R Foundation for Statistical Computing, Vienna, Austria; available at http://www.Rproject.org or R Development core Team, Vienna, Austria).

\section{Results}

A total of 167 eyes were primarily enrolled. The sample was divided into two groups depending on the surgical technique used: FLACS group including a total of 105 eyes (FLACS I and FLACS II subgroups), and conventional phacoemulsification (MANUAL) group, including a total of 62 eyes. In phase I, patients were assigned either to FLACS-I $(n=63)$ or MANUAL $(n=62)$. One of the three surgeons operated the LensX®, another perform the FLACS surgery (FLACS I) in one operating room (OR1), while the third surgeon performed the conventional phacoemulsification procedure (MANUAL) in another operating room (OR2). On subsequent days, rotated between LensX®, FLACS and MANUAL surgery (Figure 1). In the second phase (FLACS-II), all the surgeries were FLACS $(n=42)$. One of the three surgeons operated the Lens $X ®$, while the other two surgeons completed the procedures in two different ORs. Surgeons rotated in subsequent days. (Figure 2).

From the 167 eyes enrolled, six patients did not show up and one FLACS had to be reconverted to conventional phacoemulsification. Excluding the missing and incomplete data, the following patients were included for statistical analysis: 57 patients of MANUAL, 27 patients of FLACS I (the surgical time 
recorded in 32 patients was disregarded due to a protocol deviation due to the use of Verion ${ }^{\circledR}$ system [Alcon ${ }^{\circledR}$ Fort Worth, Texas, USA] between the laser procedure and the end of the procedure in the OR) and 30 patients of FLACS II (Table 1). Tables 2 to 4 display the main outcomes obtained in the two phases of the current study.

Regarding the comparison of FLACS I vs MANUAL (Table 2), both groups were comparable in terms of patient age $(p=0.85)$. Likewise, no statistically significant differences in CDE were found between groups, although less energy was used in the FLACS I subgroup (10.52 [4.84] vs 12.93 [7.27], $p=0.27$ ). This was expected as part of the "chopping" of the nucleus was performed with the femtosecond laser. Preparation time (time elapsed from the arrival of the patient to the surgical premises until the entrance to the laser or operating room) in FLACS I subgroup was statistically significantly lower compared to MANUAL group $(p<0.001)$. Total time $(p=0.60)$ and phacoemulsification time $(p=0.78)$ did not differ significantly between FLACS and MANUAL.

Regarding the comparison of FLACS II vs MANUAL (Table 3), both groups were also comparable in terms of patient age $(p=0.20)$. No statistically significant differences between groups were found in CDE $(p=0.18)$. Preparation time (time elapsed from the arrival of the patient to the surgical premises until the entrance to the laser or operating room); was statistically significantly lower $(p<0.001)$ in FLACS II subgroup compared to MANUAL group. In contrast, Total time did not differ significantly between groups $(p=0.99)$. Surgical time (defined as time from the insertion of blepharostat to its removal after ending the surgical procedure) was significantly lower in the MANUAL group compared to FLACS II subgroup $(p=0.005)$.

Finally, the comparison of FLACS I vs FLACS II (Table 4) revealed that both groups were also comparable in terms of patient age $(p=0.39)$. The CDE used in FLACS II was significantly higher than in FLACS I subgroup ( $p=0.01$ ), whereas femtosecond laser time procedure was significantly lower in FLACS II subgroup $(p<0.001)$. Preparation time $(p=0.07)$ and preparation plus laser time $(p=0.56)$ did not differ significantly between subgroups. In contrast, circulation time between the laser room and the operation room where the phacoemulsification was performed was significantly shorter in FLACS II compared to FLACS I subgroup $(p=0.008)$. Similarly, whereas femtosecond laser time procedure (the time elapsed from the moment the patient entered the OR room where the femtosecond laser procedure was conducted to the time the patient left having completed the procedure) was significantly lower in FLACS II subgroup $(p<0.001)$. Total time between both groups did not reach significant difference $(p=0.71)$. In contrast, surgical time (defined as time from the insertion of blepharostat to its removal after ending the surgical procedure) was shorter in FLACS I subgroup compared to FLACS II $(p=0.04)$. No statistically significant differences were detected in intraoperative phacoemulsification time (total time elapsed since the patient enters the OR in which the phacoemulsification was performed and exits to the recovery room) between both subgroups $(p=0.99)$.

Complications.In FLACS group, the following complications were described: incomplete capsulorrhexis (1 eye in FLACS I and 1 eye in FLACS II), not completely opened incisions (4 eyes in FLACS I and 2 eyes in 
FLACS II), capsular rupture (2 eyes in FLACS I), one reconversion to conventional phacoemulsification in FLACS I and one suspended surgery also in FLACS I. In MANUAL group the following complications were reported: poor mydriasis being needed the use iris hooks ( 1 eye) and zonular disinsertion (1 eye). All complications were successfully managed and no permanent damage occurred in these eyes. No statistically significant differences between FLACS and MANUAL groups were found in the complication rate $(p=0.56)$.

\section{Discussion}

In the last years, the safety, accuracy and reproducibility of FLACS has been widely reported in a great variety of studies. ${ }^{5-7}$ Most of the results published have been obtained in private centers, but in recent years, growing scientific evidence of the use of FLACS in public hospitals and how its introduction may affect to the efficiency of the public system is being published. ${ }^{26-28}$ Besides the evaluation of the safety of FLACS and its outcomes, the aim of the current research project was to evaluate the impact of the use of FLACS on the efficiency of a public health center with a large volume of surgeries ( 21 cataracts per day, on average) according to the design of intraoperative room patient circulation.

Unlike other reports ${ }^{5,30,31}$, in our sample FLACS procedure did not take longer than conventional phacoemulsification. In our study, "total time", defined as the time from the moment the patient enters the surgical area to the patient is discharged once the intervention is completed, was not significantly different between our groups of study, MANUAL vs. FLACS (FLACS I + FLACS II subgroups). Moreover, the circulation time between the moment after completing the laser procedure and the moment when the patient entered the OR to complete the cataract surgery was significantly shorter in FLACS II. The comparison between FLACS I and FLACS II subgroups revealed that an optimized workflow decreased the roll over and circulation times, despite performing twice the number of femtosecond surgeries in FLACS II subgroup. The preparation times were similar in both FLACS I and FLACS II subgroups, but significantly superior to the time needed in the MANUAL group.

Bascaran $^{28}$ et al. in a Spanish public setting also found that FLACS procedure did not take longer than conventional phacoemulsification. However, Ibrahim et al ${ }^{26}$ reported that the addition of FLACS in public clinical setting resulted in longer time per case than traditional cataract surgery and they also measured the "total time" of performing a FLACS surgery, including the transportation and roll-over times. However, these authors stated that although there was statistically significant difference between FLACS and manual phacoemulsification, the results may not be indicative of practical differences. ${ }^{27}$ Vasquez-Perez and colleagues ${ }^{27}$ reported in another study conducted in public clinical setting that a mobile FLACS system of a different technology than that used in our study and housed in the same operating room increased the surgical duration by 5.2 minutes.

Our center is public funded, so the regular seven cataracts per OR per surgical turn had to be maintained. Therefore, an efficient workflow had to be designed and as a consequence, FLACS intervention was not more time consuming than conventional phacoemulsification in our study. We believe that our data can 
be mainly explained by the careful control of patients' circulation in the OR. Specifically, patients started LenS $x^{\circledR}$ procedure half an hour earlier, being placed immediately afterwards at entrance of the OR, where the cataract surgery was going to be completed. It should be mentioned that the contribution of a highly motivated auxiliary staff was also very important for achieving the optimal circulation of the patient in the surgical area. Another factor that may have influenced our outcomes was the learning curve effect, with more experience and more optimization of the surgical technique as the surgeon performed more FLACS procedures. The maintenance of the regular number of surgeries after the FLACS learning curve was also reported by Ibrahim et al. ${ }^{26}$ In any case, the development of an optimized design of the workflow is crucial for increasing the efficiency of the FLACS surgical procedures in public clinical setting. Van Vliet and colleagues ${ }^{32}$ stated that the environmental context and operational focus primarily influenced process design of the cataract pathways. These authors concluded that multi-skilled team and pull planning were two crucial factors to consider when optimizing processes to increase efficiency in high-volume cataract pathways. ${ }^{32}$ Regge et $\mathrm{al}^{33}$ also confirmed that the use of specialized scrub nurses and the eye clinic design benefit flow efficiency of the cataract surgery process in hospitals. More research is still needed to determine the workflow designs that are the most adequate to obtain the maximum level of optimization of cataract surgery in public clinical settings.

Phacoemulsification procedure time was not significantly different between FLACS I subgroup and MANUAL group. Possibly, this was due to the learning curve of surgeons in FLACS I subgroup as the learning curve effect has been demonstrated to increase the surgical time. ${ }^{34}$ Likewise, the time needed to perform the phacoemulsification procedure was significantly lower in MANUAL group and FLACS I subgroup compared to FLACS II subgroup. This is consistent with previous studies showing laser cataract surgery took longer to complete compared to conventional phacoemulsification without accounting for the time taken to complete the laser procedure itself, being this due in part to irrigationaspiration requiring more instrument maneuvers and taking longer to complete. ${ }^{29,32}$ This fact may also be explained by the fact that more complex cataracts were included in FLACS II subgroup. Previous scientific evidence on the surgical time required with FLACS vs. conventional phacoemulsification have shown contradictory outcomes, with some authors reporting comparable surgical times, ${ }^{35-37}$ whereas others concluding that FLACS was more time-consuming. ${ }^{24,26,30}$ Some factors may account for such differences among studies, such as differences in terms of cataract hardness, surgical procedures, surgeon experience or technology used.

Comparison reports on Efficient Phaco time (EPT) in FLACS compared to conventional phacoemulsification are controversial: in some authors' work did not differ significantly ${ }^{13,38,39}$, whereas others reported statistically significant differences in favor of FLACS $3,16,29,37$. In our study, FLACS subgroups and MANUAL group did not show statistically significant differences. This was possibly due to the learning curve effect in the FLACS group. However, EPT was significantly lower in FLACS I subgroup compared to FLACS II subgroup, which may be explained by the inclusion of more complex cataracts FLACS II subgroup (harder cataracts, smaller sockets and mydriasis up to $8 \mathrm{~mm}$ ). Although compared with conventional phacoemulsification, FLACS for hard nuclear cataracts has shown to 
conserve phacoemulsification power, provide a significant reduction in corneal endothelial damage, and lead to faster visual rehabilitation, ${ }^{40}$ the level of EPT required even with FLACS is higher in hard than in softer cataracts.

Finally, it should be remarked that no statistically significant differences were found between MANUAL and FLACS subgroups in terms of intraoperative complications, confirming the safety of both procedures. The minimal number of complications occurring in each group were satisfactorily resolved without affecting patients' final visual outcome.

As any clinical study, our study has some limitations that should be acknowledged. First, it is a nonrandomized study and the sample size is small, especially in FLACS I subgroup, where from the initial 63 patient that underwent the surgery, only 27 eyes could be included for the statistical analysis: the surgical time recorded in 32 patients was disregarded due to a protocol deviation due to the use of Verion ${ }^{\circledR}$ system (Alcon ${ }^{\circledR}$ Fort Worth, Texas, USA) between the laser procedure and the end of the procedure in the OR. Also, in seven patients (5 in MANUAL, 2 in FLACS I), data was not properly recorded. In FLACS II six patients did not show up, probably because this part of the study was conducted in July and patients tend to be on holidays in this part of the year (Table 1). Second, although the learning curve for FLACS is significantly shorter in ophthalmologists performing both conventional phacoemulsification and refractive surgery compared to only conventional phacoemulsification, ${ }^{24,25}$ which is the case of the three surgeons involved in the study and provided that each of them performed prior to the study an initiation trial with 25 eyes each, their learning may have influenced the outcomes. Third, it was somewhat difficult to find "equivalent" patients for all groups given the characteristics of our center, which is a public hospital with an elderly population, multiple eye diseases and an indication for cataract surgery with BCVA of 0.4 logMAR. However, we tried to minimize the impact of this effect in the second part of the study (FLACS II) by including patients with white or harder cataracts (nuclear opacities from NC4 to NC6, cortical opacities from C3 to C5 and posterior subcapsular from P3 to P5, according to the LOCSIII), smaller orbits, suboptimal mydriasis (from 6 to $8 \mathrm{~mm}$ ), white cataracts and/or Fuchs endothelial dystrophy

\section{CONCLUSIONS}

In conclusion, our study suggests that a time-efficient and suitable workflow model for FLACS can be reached considering the specific requirements and restrictions of a saturated public hospital. In our setting, a high volume of cataract procedures is managed daily, and both our target population and their cataracts are frequently non-straightforward. Even so, FLACS procedure did not take longer than conventional phacoemulsification following a detailed planning for the OR workflow. Finally, our data also suggests an improvement of surgical times in FLACS with ongoing experience, although more challenging cases were included in our FLACS II subgroup. Future comparative studies on the impact of introducing FLACS using different technologies in public hospitals should be conducted. 


\section{Abbreviations}

$\mathrm{OR}=$ operation room

FLACS $=$ Femtosecond laser-assisted cataract surgery (FLACS

$\mathrm{IOL}=$ intraocular lenses

BCVA $=$ best-corrected visual acuity

$\mathrm{CDE}=$ cumulative dissipated energy

LOCSIII t= Lens Opacities Classifications System III

EPT $=$ Efficient Phaco time

\section{Declarations}

\section{Ethics approval and consent to participate}

The present study was approved by the Ethics Committee of Hospital Regional Universitario Málaga (Carlos Haya), Spain. All patient's included signed an informed consent to participate in the study.

This study has been carried out following the Declaration of Helsinki and Good Clinical Practices guidelines. Personal data was processed according to guidelines established by the Spanish Law of Data Protection (LOPD).

Consent for publication

In the informed consent signed by the patients there was a specific mention of the possible use of the data in publications.

\section{Availability of data and material}

Data of this study are available on request.

\section{Competing interests}

David P Piñero-Llorens is a member of the Board of BMC Ophthalmology. The resto of authors have no competing interest to disclose.

\section{Funding}


Marina Rodríguez-Calvo-de-Mora,Cristina Pérez-Casaseca, Emilio Espejo-de-los-Riscos and Gustavo Fernández-Baca-Vaca G received an Investigator Initiatied Trial from Alcon. The results of this study were presented at ESCRS meeting of Vienna, September 2018, by Marina Rodríguez-Calvo-de-Mora, travel expenses were partially covered by Alcon. The authors have no conflict of interest to disclose.

\section{Authors' contributions}

Jacinto Villavilla-Castillo: Design of the study.

Marina Rodríguez-Calvo-de-MoraDesign of the study, data collection, data analysis, manuscript writing.

Cristina Pérez-Casaseca: Surgeon involved in the study, data collection.

Emilio Espejo-de-los-Riscos: Surgeon involved in the study, data collection.

Gustavo Fernández-Baca-Vaca: Surgeon involved in the study, data collection.

Ildefonso Fernández-Baca-Casares: Design of the study.

David P. Piñero-Llorens: data analysis, manuscript writing.

\section{Acknowledgements}

To our dedicated surgery staff.

To Olga Pérez, from FIMABIS (Fundación Pública Andaluza para la Investigación de Málaga en Biomedicina y Salud), for the help in statistics calculations.

\section{References}

1. Day AC, Gore DM, Bunce C, Evans JR. Laser-assisted cataract surgery versus standard ultrasound phacoemulsification cataract surgery. Cochrane Database Syst Rev 2016; 7:CD010735.

2. Foster A, Gilbert C, Johnson G. Changing patterns in global blindness: 1988-2008. Community Eye Health 2008; 21:37-39.

3. Popovic M, Campos-Möller X, Schlenker MB, Ahmed IIK. Efficacy and Safety of Femtosecond LaserAssisted Cataract Surgery Compared with Manual Surgery. Ophthalmology 2016; 123:2113-2126.

4. Hoffman RS, Fine IH, Packer M. New phacoemulsification technology. Curr Opin Ophthalmol 2005; 16:38-43. 
5. Agarwal A, Jacob S. Current and effective advantages of femto phacoemulsification. Curr Opin Ophthalmol 2017; 28:49-57.

6. Chen X, Xiao W, Ye S, Chen W, Liu Y. Efficacy and safety of femtosecond laser-assisted cataract surgery versus conventional phacoemulsification for cataract: a meta-analysis of randomized controlled trials. Sci Rep 2015; 5:13123.

7. Ranka M, Donnenfeld ED. Femtosecond laser will be the standard method for cataract extraction ten years from now. Surv Ophthalmol 2015; 60:356-60.

8. Hatch KM, Talamo JH. Laser-assisted cataract surgery: benefits and barriers. Curr Opin Ophthalmol 2014; 25:54-61.

9. Corredor-Ortega C Gonzalez-Salinas R, Montero MJ, González-Flores R, Collura-Merlier A, CervantesCoste G, Mendoza-Schuster E, Velasco-Barona C. Femtosecond laser-assisted cataract surgery in pediatric patients. J AAPOS 2018; 22:148-149.

10. Dick HB, Schultz T. Femtosecond laser-assisted cataract surgery in infants. J Cataract Refract Surg 2013; 39:665-8.

11. Mastropasqua L, Toto L, Mastropasqua A, Vecchiarino L, Mastropasqua R, Pedrotti E, Di Nicola M. Femtosecond laser versus manual clear corneal incision in cataract surgery. J Refract Surg 2014; 30:2733.

12. Abell RG, Kerr NM, Howie AR, Mustaffa Kamal MA, Allen PL, Vote BJ. Effect of femtosecond laserassisted cataract surgery on the corneal endothelium. J Cataract Refract Surg 2014; 40: 1777-1783.

13. Takacs Al, Kovács I, Miháltz K, Filkorn T, Knorz MC, Nagy ZZ. Central corneal volume and endothelial cell count following femtosecond laser-assisted refractive cataract surgery compared to conventional phacoemulsification. J Refract Surg 2012; 28: 387-391.

14. He L, Sheehy K, Culbertson W. Femtosecond laser-assisted cataract surgery. Curr Opin Ophthalmol 2011; 22: 43-52.

15. Masket S, Sarayba M, Ignacio T, Fram N. Femtosecond laser-assisted cataract incisions: architectural stability and reproducibility. J Cataract Refract Surg 2010; 36: 1048-1049.

16. Abell RG, Kerr NM, Vote BJ. Femtosecond laser-assisted cataract surgery compared with conventional cataract surgery. Clin Exp Ophthalmol 2013; 41: 455-62.

17. Conrad-Hegener I, Al Juburi M, Schultz T, Hengerer FH, Dick HB. Corneal endothelial cell loss and corneal thickness in conventional compared with femtosecond laser-assisted cataract surgery: threemonth follow-up. J Cataract Refract Surg 2013; 39: 1307-13. 
18. Nagy ZZ, Mastropasqua L, Knorz MC. The use of femtosecond lasers in cataract surgery: review of the published results with the LenSx system. J Refract Surg 2014; 30: 730-740.

19. Nagy ZZ, Kranitz K, Takacs Al, Mihaltz K, Kovacs I, Knorz MC. Comparison of intraocular lens decentration parameters after femtosecond and manual capsulotomies. J Refract Surg 2011; 27: 564569 .

20. Kranitz K, Takacs A, Mihalta K, Kovacs I, Knorz MC, Nagy ZZ. Femtosecond laser capsulotomy and manual continuous curvilinear capsulorhexis parameters and their effects on intraocular lens centration. J Refract Surg 2011; 27: 558-563.

21. Friedman NJ, Palanker DV, Schuele G, Andersen D, Marcellino G, Seibel BS, Batlle J, Feliz R, Talamo JH, Blumenkranz MS, Culbertson WW. Femtosecond laser capsulotomy. J Cataract Refract Surg 2011; 37: 1189-1198.

22. Trigueros JA, Piñero DP, Ismail MM. Profitability analysis of a femtosecond laser system for cataract surgery using a fuzzy logic approach. Int J Ophthalmol 2016; 9: 1046-50.

23. Abell RG, Vote BJ. Cost-effectiveness of femtosecond laser-assisted cataract surgery versus phacoemulsification cataract surgery. Ophthalmology 2014; 121: 10-16.

24. Bali SJ, Hidge C, Lawless M, Toberts TV, Sutton G. Early experience with the femtosecond laser for cataract surgery. Ophthalmology 2012; 119: 891-899.

25. Roberts TV, Lawless M, Bali SJ, Hodge C, Sutton G. Surgical outcomes and safety of femtosecond laser cataract surgery. A prospective study of 1500 consecutive cases. Ophthalmology 2013; 120: 227233.

26. Ibrahim T, Goernert P, Rocha G. Effect of femtosecond laser on efficiency of cataract surgery in public setting. Can J Ophthalmol 2018; 53: 56-59.

27. Vasquez-Perez A, Simpson A, Nanavaty MA. Femtosecond laser-assisted cataract surgery in a public teaching hospital setting. BMC Ophthalmol 2018; 18: 26.

28. Bascaran L, Alberdi T, Matinez-Soroa I, Sarasqueta C, Mendicute J. Differences in energy and corneal endothelial beyween Femtosecond laser-assisted and conventional cataract surgery: prospective, individual, randomized controlled trial. Int J Ophthalmol. 2018;11(8):1308-1316.

29. Ho T, Fan R, Hong WW, Khian KB. Maximal mydriasis evaluation in cataract surgery. Indian Journal of Ophthalmology, 1992;40(3).74-78.

30. Lubahn JG, Donaldson KE, Culbertson WW, Yoo SH. Operating times of experienced cataract surgeons beginning femtosecond laser-assisted cataract surgery. J Cataract Refract Surg 2014; 40: 1773-6. 
31. Day AC, Smith PR, Tang HL, Aiello F, Hussain B, Maurino V, Marshall J, Saleh GM. Surgical efficiency in femtosecond laser cataract surgery compared with phacoemulsification cataract surgery: a casecontrol study. BMJ Open 2018 Feb; 8: e018478.

32. Van Vliet EJ, Bredenhoff E, Sermeus W, Kop LM, Sol JC, Van Harten WH. Exploring the relation between process design and efficiency in high-volume cataract pathways from a lean thinking perspective. Int J Qual Health Care 2011; 23: 83-93.

33. De Regge M, Gemmel P, Duyck P, Claerhout I. A multilevel analysis of factors influencing the flow efficiency of the cataract surgery process in hospitals. Acta Ophthalmol 2016; 94: 31-40.

34. Grewal DS, Dalal RR, Jun S, Chou J, Basti S. Impact of the learning curve on intraoperative surgical time in femtosecond laser-assisted cataract surgery. J Refract Surg 2016; 32: 311-7.

35. Schargus M, Suckert N, Schultz T, Kakkassery V, Dick BH. Femtosecond laser-assisted cataract surgery without OVD: a prospective intraindividual comparison. J Refract Surg 2015; 31: 146-52.

36. Yu AY, Ni LY, Wang QM, Huang F, Zhu SQ, Zheng LY, Su YF. Preliminary clinical investigation of cataract surgery with a noncontact femtosecond laser system. Lasers Surg Med 2015; 47: 698-703.

37. Conrad-Hengerer I, Hengerer FH, Schultz T, Dick HB. Effect of femtosecond laser fragmentation of the nucleus with different softening grid sizes on effective phaco time in cataract surgery. $\mathrm{J}$ Cataract Refract Surg 2012; 38: 1888-94.

38. Ecsedy M, Milhatz K, Kovacs A, Filkom T, Naggy ZZ. Effect of femtosecond laser cataract surgery in the macula. J Refract Surg 2011;27(10):717-722.

39. Murchs-Edlmay et al. Intraocular comparison between femtosecond laser-assisted and conventional cataract surgery. J Cataract Refract Surg. 2017;42(2):215-222.

40. Chen X, Yu Y, Song X, Zhu Y, Wang W, Yao K. Clinical outcomes of femtosecond laser-assisted cataract surgery versus conventional phacoemulsification surgery for hard nuclear cataracts. J Cataract Refract Surg 2017; 43: 486-91.

\section{Tables}




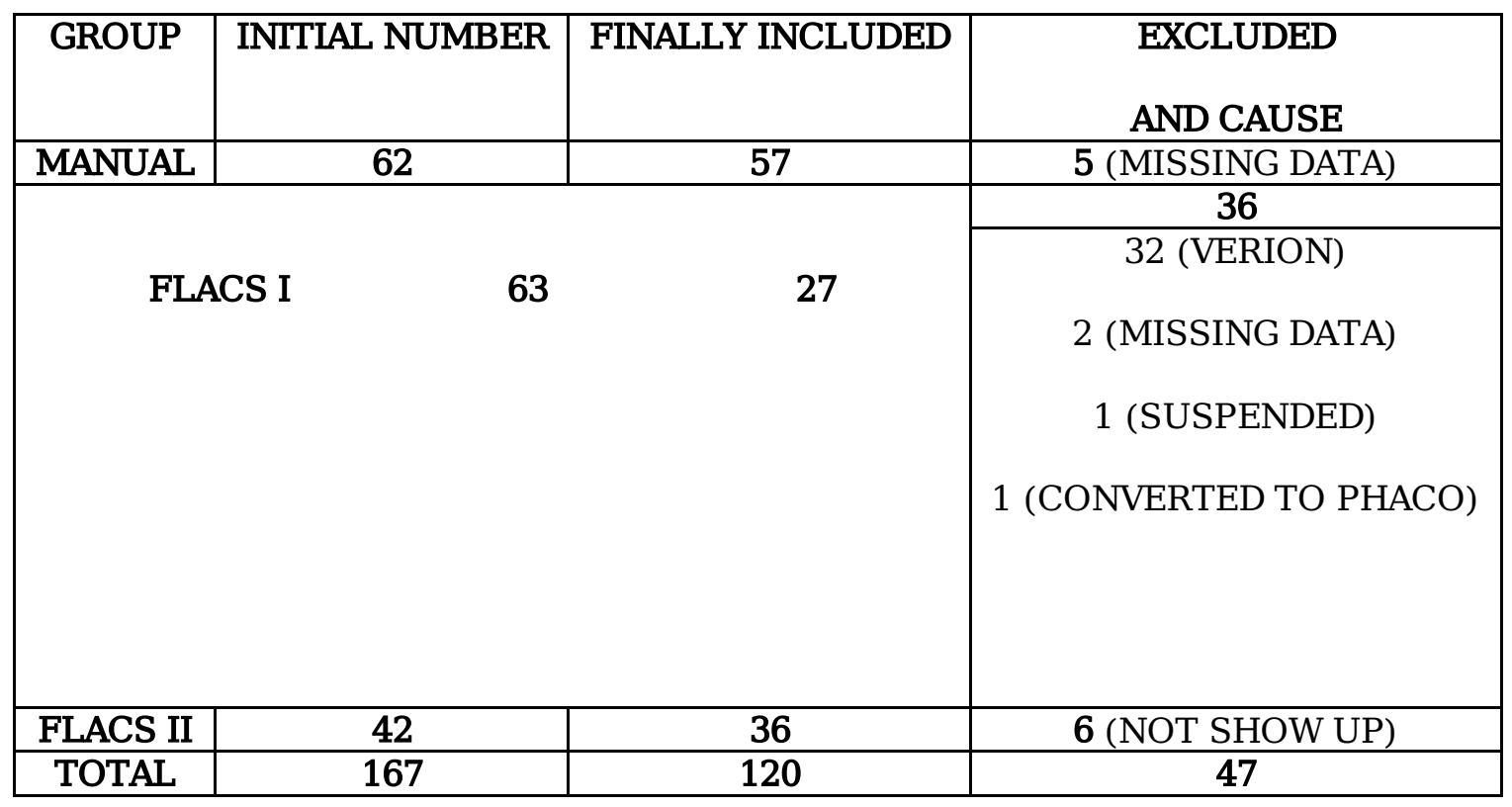

Table 1. Patients included and excluded from the study and causes of exclusion. MANUAL = conventional phacoemulsification; FLACS I: femtosecond laser assisted cataract surgery, subgroup I; FLACS II: femtosecond laser assisted cataract surgery, subgroup II; Verion ${ }^{\circledR}$ : the surgical time recorded in 32 patients was disregarded due to a protocol deviation due to the use of system (Alcon ${ }^{\circledR}$ Fort Worth, Texas, USA) in FLACS I. 


\begin{tabular}{|c|c|c|c|}
\hline Variable & FLACS I & MANUAL & p-value \\
$\mathbf{N}=\mathbf{2 7}$ & $\mathbf{N}=62$ & \\
\hline Age & $68.92(11.74)$ & $69.10(10.57)$ & $0.85^{\mathrm{b}}$ \\
\hline CDE & $10.52(4.84)$ & $12.93(7.27)$ & $0.27^{\mathrm{b}}$ \\
\hline Preparation Time & $26.11(10.64)$ & $47.82(15.24)$ & $<0.001^{\mathrm{a}}$ \\
\hline Intraoperative Phacomulsification Time & $22.66(3.94)$ & $18.5(9.46)$ & $0.07^{\mathrm{b}}$ \\
\hline Total Surgical Time & $72.74(14.63)$ & $70.88(16.54)$ & $0.6^{\mathrm{b}}$ \\
\hline
\end{tabular}

Table 2.- Comparison between MANUAL group and FLACS I subgroup. Each variable is represented by mean and standard deviation: Mean (Standard Deviation); a: T-student independent group, b: MannWhitney test. CDE = Cumulative Dissipated Energy; Preparation Time (time from the arrival of the patient to the surgical premises until the entrance to the laser o operating room); Cataract Surgery Time (time from the insertion of blepharostat to its removal after ending the surgical procedure); Intraoperative Phacomulsification Time (time from the moment the patient entered the OR to the moment the patient left the OR after finishing the surgery), Total Surgical Time (and time from the admission of the patient to the surgical area to the discharge once the intervention was finished). All surgical and rollover times are measured in minutes and seconds. 


\begin{tabular}{|c|c|c|c|}
\hline Variable & $\begin{array}{c}\text { FLACS II } \\
\mathbf{N}=\mathbf{3 6}\end{array}$ & $\begin{array}{c}\text { MANUAL } \\
\mathbf{N}=62\end{array}$ & p-value \\
\hline Age & $71.92(8.44)$ & $69.11(10.57)$ & $0.2^{\mathrm{a}}$ \\
\hline CDE & $14.40(6.7)$ & $12.93(7.27)$ & $0.18 \mathrm{~b}$ \\
\hline Preparation Time & $31.6(11.87)$ & $47.82(15.24)$ & $<0.001^{\mathrm{a}}$ \\
\hline Total Cataract Surgery Time & $12.64(5.69)$ & $9.98(3.99)$ & $\mathbf{0 . 0 0 5 ^ { \mathrm { b } }}$ \\
\hline Intraoperative Phacomulsification Time & $22.79(4.65)$ & $18.58(9.46)$ & $0.06^{\mathrm{b}}$ \\
\hline Total Surgical Time & $70.91(12.69)$ & $70.88(16.54)$ & $0.99^{\mathrm{a}}$ \\
\hline
\end{tabular}

Table 3.- Comparison between MANUAL group and FLACS I subgroup. Each variable is represented by mean and standard deviation: Mean (Standard Deviation); a: T-student independent group, b: Mann-Whitney test. $\mathrm{CDE}=$ Cumulative Dissipated Energy; Preparation Time (time from the arrival of the patient to the surgical premises until the entrance to the laser o operating room); Cataract Surgery Time (time from the insertion of blepharostat to its removal after ending the surgical procedure); Intraoperative Phacomulsification Time (time 
from the moment the patient entered the OR to the moment the patient left the OR after finishing the surgery),

Total Surgical Time (and time from the admission of the patient to the surgical area to the discharge once the intervention was finished). All surgical and rollover times are measured in minutes and seconds.

\begin{tabular}{|c|c|c|c|}
\hline Variable & FLACS I & FLACS II & p-value \\
\hline Age & N = 36 & \\
\hline CDE & $68.92(11.74)$ & $71.92(8.44)$ & $0.39^{\mathrm{b}}$ \\
\hline Preparation time & $10.52(4.83)$ & $14.4(6.7)$ & $0.01^{\mathrm{a}}$ \\
\hline Laser time & $26.11(10.64)$ & $31.6(11.87)$ & $0.07^{\mathrm{a}}$ \\
\hline Preparation + laser time & $8.96(3.22)$ & $5.26(1.54)$ & $<0.001^{\mathrm{b}}$ \\
\hline End of Laser to Beginning of Phacomulsification & $15.03(11.96)$ & $7.96(5.83)$ & $\mathbf{0 . 0 0 8 ^ { \mathrm { b } }}$ \\
\hline Total Cataract Surgery Time & $10.48(2.5)$ & $12.65(5.69)$ & $\mathbf{0 . 0 4}{ }^{\mathrm{b}}$ \\
\hline Intraoperative Phacomulsification & $22.66(3.94)$ & $22.79(4.65)$ & $0.99^{\mathrm{b}}$ \\
\hline Total Surgical Time & $72.74(14.62)$ & $70.91(12.68)$ & $0.71^{\mathrm{b}}$ \\
\hline
\end{tabular}


Table 4.- Comparison between FLACS I and FLACS II subgroups. Each variable is represented by mean and standard deviation: Mean (Standard Deviation); a: T-student independent group, b: Mann-Whitney test. CDE = Cumulative Dissipated Energy; Preparation Time (time from the arrival of the patient to the surgical premises until the entrance to the laser o operating room); Laser time (time elapsed from the moment the patient entered the OR room where the femtosecond laser procedure was conducted to the time the patient left having completed the procedure); Preparation time + Laser time (time from the arrival of the patient to the surgical premises until the entrance to the laser operating room plus time of the LenSx procedure); End of Laser to Beggining of Phacomulsification (time elapsed from the end of the LenSx procedure to the entrance in the operation room); Cataract Surgery Time (time from the insertion of blepharostat to its removal after ending the 
surgical procedure); Intraoperative Phacomulsification Time (time from the moment the patient entered the OR to the moment the patient left the OR after finishing the surgery), Total Surgical Time (and time from the admission of the patient to the surgical area to the discharge once the intervention was finished). All surgical and rollover times are measured in minutes and seconds.

\section{Image Legends}

Image 1.- LenSx® screen caption. Advanced cataract.

Image 2.- LenSx® screen caption. Advanced cataract and small pupil.

Image 3.- LenSx® screen caption. White cataract.

\section{Figures}

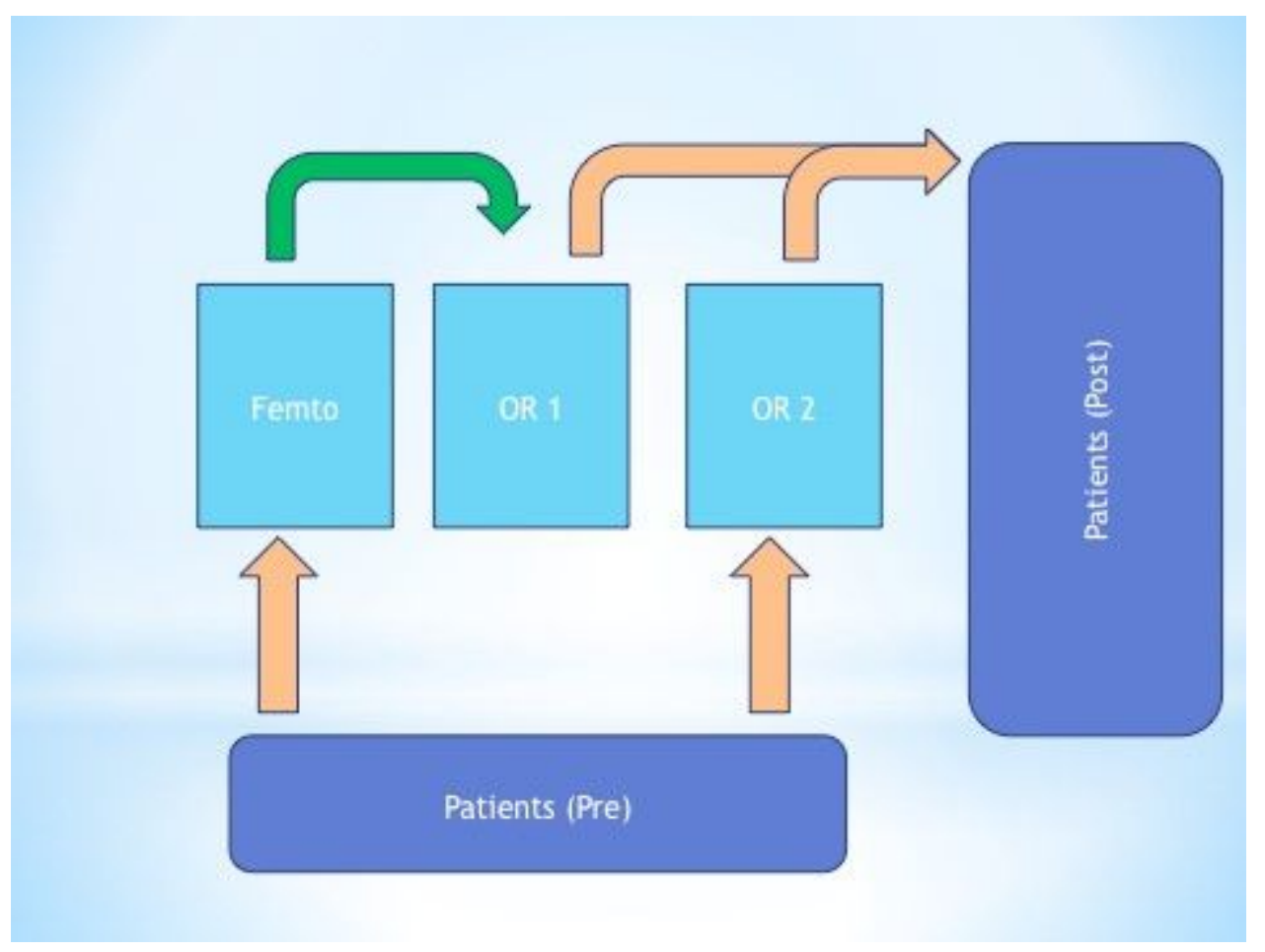

\section{Figure 1}

Workflow of patients in the phase 1 of the study. Abbreviations: OR, operating room. 


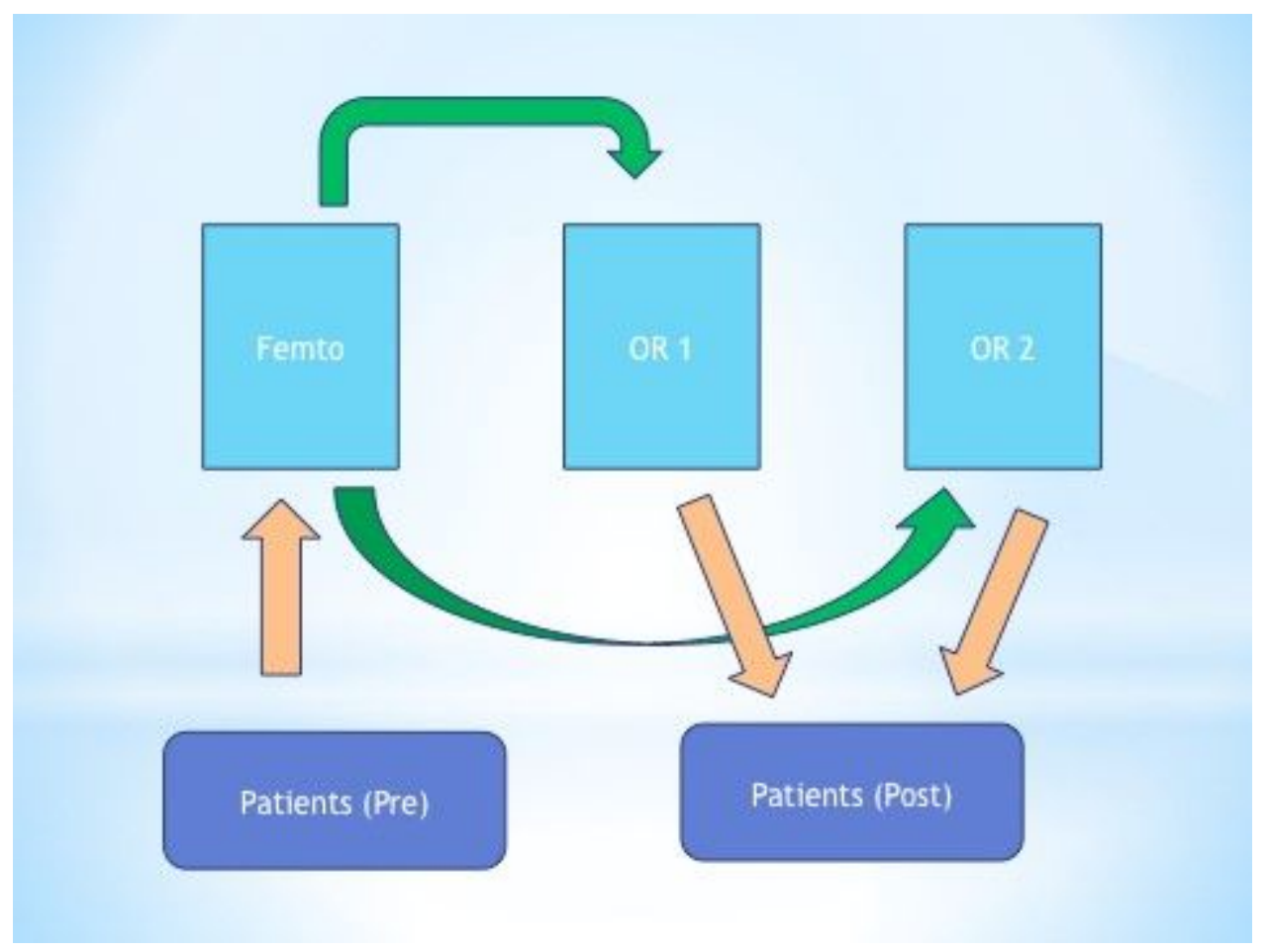

\section{Figure 2}

Workflow of patients in the phase 2 of the study. Abbreviations: OR, operating room.

\section{Supplementary Files}

This is a list of supplementary files associated with this preprint. Click to download.

- Image3whitecataract.jpg

- Image2smallpupil.jpg

- Image1hardcataract.jpg 